\title{
Multi-Agent Wireless System for Dynamic and Local Combined Pricing, Allocation and Billing
}

\author{
Clemens Kloeck, Holger Jaekel and Friedrich K. Jondral \\ Institut fuer Nachrichtentechnik, Universitaet Karlsruhe (TH) Germany
}

$\{$ kloeck,jaekel,fj\}@int.uni-karlsruhe.de

\begin{abstract}
In established communication systems prices are determined in a quasi-static way and for a large area according to a fixed price model. Additionally, the main decision criteria of resource allocation is the arrival time of resource requests. This system approach is not able to respond to the spatially distributed and dynamic users' demand and willingness-to-pay. In this paper a system will be proposed to combine pricing, allocation and billing in order to react dynamically and locally to the market assuming that the user terminal has cognitive radio abilities and multiple interfaces.
\end{abstract}

Index Terms-Dynamic and Distributed Resource Allocation, Auction Theory, Cognitive Radio, Multi-homing, Artificial Intelligence, Multi-Agent System, Communication System

\section{INTRODUCTION}

In established communications systems with an underlying provider infrastructure the market is designed according to the Fixed Price Model (FPM). A user can get access to the network only if there are free resources controlled by the Base Station (BS) within a cell. Furthermore, he has to accept the fixed price for a wide area and quasi-static in time. The user has to pay the same price whether there is a high or a low demand in the cell. Thus, if his preferences and purchase power allow him to spend more money for using Radio Resource Goods (RRG), he is not able to influence the allocation. For the same reason the operator misses the chance to increase his monetary gain by adapting the price for RRG to the users' RRG evaluation.

To overcome these problems, this paper will introduce a distributed, dynamical and combined pricing, allocation and billing system [1], suitable for wireless infrastructure communications systems which are capable to manage multi-homing. By applying Cognitive Radio (CR) abilities not only to the allocation but also to this combined architecture, it is mandatory to dynamically allocate RRG by an Auction Sequence (AS) to exploit the CR abilities [2]. The repetition of auctions should happen very fast up to milliseconds to react on the load dynamic. A class of auctions, the multi-unit sealed-bid auction, is suitable to execute the auction within specified time and with an acceptable signaling effort in comparison to sequential auctions [3].

Based on "Dynamic and Local Combined Pricing, Allocation and Billing System with Cognitive Radios", by Kloeck C., Jaekel H. and Jondral F. K. which appeared in the Proceedings of the IEEE DySPAN 2005, Baltimore, USA, September 2005. (c)2005 IEEE
After refering to related work in Section II, in Section III it will be proven that the extension of CR abilities to the combined functionality of pricing, allocation and billing and its exploitation requires AS for allocation. The application of the CR is extended to several different environments, whereas its abstraction is discussed in Section IV. In Section V the RRG properties are determined assuming multi-homing. Afterwards, a system which is capable to execute the AS under the CR assumption is described in Section VI and simulation results are discussed in Section VII.

\section{RELATED WORK}

In communication networks, the access and allocation mechanisms can be divided into deterministic and statistical procedures. ALOHA and the time discrete version slotted ALOHA represent the latter case. Deterministic mechanisms possess the advantage, that both access and allocation are determined and thus the interference is reduced. Besides CSMA/CA and CSMA/CD [4], mechanisms were proposed based on repeated auctions. The so-called Resource Auction Multiple Access (RAMA) [3],[5],[6] periodically auctions access credits. This protocol is based on sequential single-unit auctions and bids that are randomly chosen. In this context the user cannot use the bids to express his needs or convey any information. Furthermore, the auction procedure can be more efficient in terms of time and signaling by applying multiunit sealed-bid auction as proposed in [2]. D-RAMA [7] removes the randomness of the bids and allows the UT to express the buffer sizes by the bid values. The main idea is to improve the Quality of Service (QoS), to divide the resources depending on the buffer sizes and the QoS parameters.

The aforementioned protocols do not allow the users to incorporate the purchase power and the preferences for the different QoS classes, the experience of past auctions and future events. In this paper, an auction sequence mechanism and a system will be presented which extends the past work by allowing the users to express their urgency, needs, purchase power and preferences combined with a QoS-aware buffer management.

Besides the access and allocation mechanism, the exploitation of $\mathrm{CR}$ abilities enhance the system dynamic, because a communication cell converges to a Multi Agent System (MAS). Especially, in the fields of resource allo- 
cation, utility functions, language efficiency and envyfreeness have to be considered.

The economical field auction theory mainly deals with single shot auction, but not with auction repetition. The framework of the optimal multi-unit auction is proposed in [8], but does not deal with repetitions and does not include learning facilities of the UTs. Gaining experience of the past and applying it in the current auction destroy the independence of the single shot auctions.

\section{Auction Sequences for Resource ALLOCATION}

The system presented in Section VI will offer the RRG locally and dynamically in time to respond to the demand changes in space and time. Considering hotspots like congresses or soccer events, which occur only for a certain time and in a certain area covered by at most a few BSs, the RRG of these BS will be highly demanded, whereas, if users move away, the RRG of the other BSs will then be wanted. This moving hotspots show a highly dynamical demand graph in space and in time. Therefore, the RRG of each BS should be offered to the users attended in the cell and the price depends on the demand of the proper cell.

If the operator wants to maximize his gain, he has to charge each user by the maximum price the user is willing to pay. In order to find this upper border, a negotiation has to take place. On the other hand, the user can incorporate his preferences and purchase power into the negotiation to get access to the network, in comparison to FPM.

Because the demand changes very fast, agents located in the MAC of the BS and the User Terminal (UT) side will negotiate among each other. These agents are algorithms which need a proper protocol and rules to negotiate. A negotiation following proper instructions, which can be implemented in a predefined algorithm, is referred to as the auction.

Considering FPM, the UT needs not to be intelligent with respect to pricing, allocation and billing, because the price is fixed and the BS assigns RRG to the UT, if available, regardless of the other users. On the other hand, by an underlying auction sequence, the UT can use experiences gained in past auctions in order to estimate the behavior of the other UTs and to modify its bidding strategy. This requires the ability to learn about the EnVironment (EV) and to modify the action in order to optimize its utility. Both, gaining cognition and acting accordingly, are part of the definition of a CR. Therefore, maximizing of user's utility mandatorily requires $\mathrm{CR}$ abilities in a distributed pricing, allocation and billing system.

\section{Cognitive Radio in A}

\section{TECHNOLOGICAL-ECONOMICAL ENVIRONMENT}

A CR is basically defined as an intelligent entity which is sophistically seeking for bandwidth in order to set up a communication connection [9]. In this section, the CR definition will be extended to a general usage in arbitrary SUBEnVironments (SUBEVs).
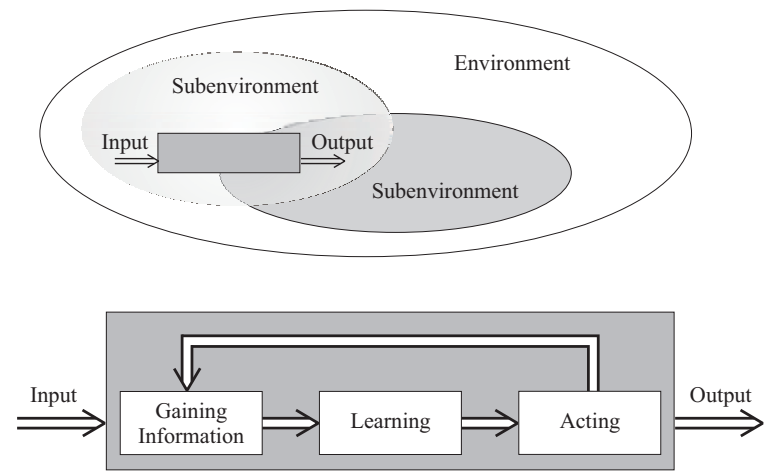

Fig. 1. Cognitive Radio in subenvironments

\section{A. Definition of Subenvironments}

The awareness of a CR can be abstracted in a way that the CR acts in a specific SUBEV. A SUBEV is a subset of the whole EV. Examples can be a technical $\mathrm{EV}$ to detect occupied spectrum as defined by the FCC [9]. For example, this SUBEV could consist of the FFT information of the spectrum, the SNR and information of possible spectrum occupation duration and shapes of different Radio Access Technologies (RATs). The SUBEVs need not to be disjoint, i.e., they can overlap. The SUBEV which is tackled by the AS consists of 1) operator's and 2) user's behavior, 3) technical, 4) physical and 5) economical SUBEVs:

1) Operator's behavior is mainly focused on optimizing his monetary gain, offering and charging services.

2) User's behavior can be represented by his preferences, purchase power and the action characteristics which can be categorized in risk-neutral, risk-averse and risk-encouraged depending on the other subenvironments.

3) The technical environment includes the demand occurring from the data a user wants to sent and the characteristics of the RATs available.

4) The channel influences the data transmission and can be described by SNR and SNIR, respectively.

5) The economical aspects include the purchase power, reaction, number and demand of the competitors, the outcomes of the auctions, the reserve prices, the offered RRG, etc.

\section{B. Cognitive Radio within a Subenvironment}

The abilities of a CR can be mainly categorized in three functions: Gaining Information (GI), Learning Process (LP) and Acting Function (AF) as depicted in Figure 1. The GI extracts information out of the incoming SUBEVs parameters and provides the information to LP. Based on past and current information the LP makes specific conclusions with respect to preferences and utility functions. The AF receives the conclusions of the LP and does not necessarily act in the same SUBEV. AF can also influence more than one SUBEV, e.g., the CR does not act in the technical EV mainly limited to the spectrum, but also influences the economical $\mathrm{EV}$ with its decision to use a proper bandwidth. 


\section{Cognitive Radio applying to Auction Sequence}

The CR abilities in the Auction Sequence EnVironment (ASEV) have to be divided into the network side, that is the BS, and the user side, that is the UT. The Economic Manager (EM) including the CR functions is responsible for the auction process in the MAC of the BS. On the other hand the RRG Auction Agent (RAA) represents the user's demand and interests within the auction and is also equipped with CR abilities.

The ASEV comprises the interests of the operator and users, the physical conditions of the data transmission and the technical and economical aspects. The ASEV can be further divided into SUBEVs:Neither the users nor the operators can detect the whole ASEV, since they do not have access to the complete information data. Furthermore, assuming an identical ASEV for different users [10], the different GIs do not necessarily extract the same information. Moreover, some data and parameters of the ASEV are only known to one RAT and thus are private information. This leads to different information provided to the different GIs, whereas there is also common information like the reserve price $r$ and the maximum RRG $N_{\max }$ offered per RAT. One major task of the LP is to estimate the behavior of the other users in order to conclude the most opportune action following game theory [10]. The AF transforms the conclusion into an action and, especially in the sense of the aforementioned ASEV, these actions will afterwards affect the same SUBEV, thus creating a recursive behavior.

\section{INFLUENCE OF THE RRG to THE MULTI-HOMING SYSTEM STRUCTURE}

The auction repetition can be both periodically and spontaneously. Based on the underlying periodical repetition of logical control protocols in communication systems, like TDMA systems, the implementation of fast auction repetitions up to frame-based auctioning is more suitable than undetermined auction sequences. Moreover, the probability of a certain number of participants attending the spontaneous auction is lower than the one of periodical auctions. In the following only periodical auction repetition is considered.

Generally, a seller can only offer a good if he can sell it for sure. Applying this to the auction mechanism and first assuming the user cannot perform multi-homing, this mechanism can only offer goods which are not depending on the user's environment. E.g., if the EM offers data or data rate and the user enters a radio shadow, the offered good cannot be provided by the operator due to bad SNR. Thus, the RRG can be frequency time bits in an FDMA/TDMA system, code time bits in an CDMA system like UMTS or time bits in a TDMA system like WLAN IEEE 802.11a.

As a second possibility, the user is able to send the data over more than one RAT simultaneously, that is its UT can get data by multi-homing. For the sake of simplicity, assuming that multi-homing occurs only by RATs of one operator, it seems a UT can bid for data or data rate no matter over which RATs the operator will transmit the data. But, following the same argumentation as before, even in this case, the operator can only offer RRG. The RRG of different RATs need not mandatory to be the same and keeping in mind that each RAT is only specialized for a few services and not all UTs are able to transmit over all RATs, the demand per specific service and resources available can only result in a competition, if the UTs bid for the RRG of each RAT solely. Nevertheless, the traffic splitting over the RRG of the RATs, accessible to the user, data traffic can be optimized by a Joint Radio Resource Management (JRRM) [11]. It can also be envisaged that if a user won RRG of a RAT and the connection becomes worse, the JRRM exchanges parts or the whole RRG of another RAT if available and UT possesses an interface in order to increase the QoS. Therefore, the UT bids for RRG of each RAT available and gets a certain number of General Resource Elementary Credits (GRECs) per RAT, kind of vouchers, which are exchangeable if increasing the QoS of the UT and not decreasing the revenue of the operator.

\section{SYSTEM DESCRIPTION}

\section{A. Overview}

The system proposal describes a spatially distributed and dynamical RRG pricing, allocation and billing thereby applying CR abilities to a UT which is capable to communicate over more than one RAT simultaneously (see Figure 2).

The UT attends to $N$ cells of $N$ different BS. Without loss of generality, it could also envisaged that both the RATs are not necessarily need to be different and the UT only attends to a subset of RATs available. The RAA is distributed within the MAC of the UT. Based on the multi-homing ability the RAA needs a convergence entity called Inter MAC (IMAC) which manages the coordination between the single MACs of the RATs in order to provide a common interface for the $3^{\text {rd }}$ layer of the OSI model. The IMAC comprises of a User Profile Manager (UPM) and an Inter Data GREC Mapping (IDGM). The user can adjust his preferences, behavior and purchase power for each QoS class through an GUI of the UPM. This data will be mapped in a set of suitable parameters and if necessary transformed for each BIdding Strategy (BIS) by the UPM. The IDGM manages the $L$ QoS buffers. Based on the parameters of each QoS class $\mathrm{QoS}_{l}$, IDGM categorizes the data in each buffer in two main categories: the critical data which should be mandatorily sent within the auction period in order to fulfill the QoS requirements and the uncritical data which can remain in the buffer a little longer without violating the QoS requirements. The IDGM maps the categorized data into RRG for each BIS of a specific RAT regarding the current reserve price $r$ of each RAT, the proper demand and the success of the proper BIS. The BIS gets the preference, behavior and purchase power parameters from the UPM, the part of GRECs needed from the IDGM as input and reserve price $r$ from the 


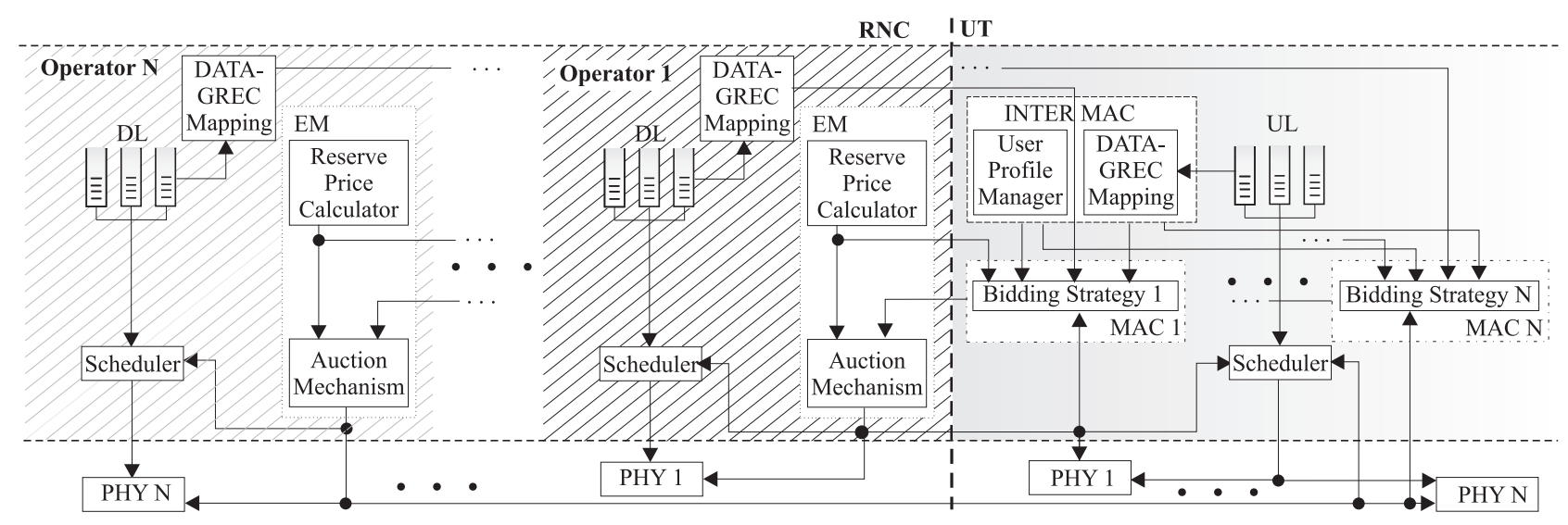

Fig. 2. Distributed allocation and pricing system

Reserve Price Calculator (RPC) as input. Furthermore, the results of past auctions may be used to calculate the bidding vector in order to minimize the difference of the utility expected to the one wanted.

Each BS possesses an EM which is responsible for the auction process. Its main two functionalities are included in the two subentities RPC and Auction Mechanism (AM) in which the CR abilities are spread. The RPC is the leverage of the operator in the auction process, because each bid has to exceed the reserve price $r$. Thus, the RPC adapts $r$ dynamically to the market in order to increase the operator's gain. The AM must be known to all participants in order to calculate the proper bids. All the RAAs which want to participate at the auction submit their bid vector to the AM. The AM chooses its bids and assigns them GRECs in order to maximize the operator's gain regarding the technical characteristic of the underlying RAT. In the allocation vector, the number of GRECs won, the proper price, link, QoS class and UT IDentification (UTID) are transmitted. Depending on the AM, the information of other UTs are partially or not at all available for a UT. The more information an RAA gets of the others, the better it can estimate the behavior of the competitors. The allocation vector is also conveyed to the MAC scheduler and the PHY, both in the UL and the DL to read out the data of the buffers and adjust the transmission modules.

\section{B. RRG Auction Agent}

The aim of the RAA is to satisfy the user's needs, because in this system the user can mainly influence and is responsible for the fulfillment of the QoS depending on his purchase power and preferences. This responsibility has been shifted from the network side in past and current system to the user side in this system description regarding the auction mechanism and the CR awareness. Nevertheless, the network side can also offer functionalities which improve the QoS as the JRRM [11], but this can be seen as an additional service.

Normally, the bidding strategy should be an overall function in which a common optimization of all bid vectors for all RATs available should take place. But this would increase the computational power and duration enormously, therefore a modular approach is presented in which the RAA functionalities are divided in the inter MAC layer and the MAC of each RAT. The inter MAC layer comprises the UPM, IDGM, QoS buffers and scheduler, whereas the BIS for each RAT is located in the proper MAC. This functionalities and their interfaces will be described below.

\section{User Profile Manager}

The UPM is the interface between the user and the RAA. The parameters a user has to adjust should not be too much regarding simplicity of service, but sufficient to characterize the user's behavior with respect to the auction. The user properties can be categorized in three main topics:

1) Preferences: For example, a user may prioritize the QoS class mainly used for phone calls. Whereas, maybe due to his bad eyes, it does not matter if the video conference images are disturbed. Therefore, the preference for the video QoS class will be lower than for the voice class. It could be envisaged only to set one preference parameter $\alpha_{l}^{x, y}$ per data category $x$, QoS class $\mathrm{QoS}_{l}$ and link $y$ of the IDGM as suggested in [2].

2) Purchase Power: The purchase power can be both, an averaged value of certain number of auctions to allow purchase peaks or a strong budget border which must be exceeded in any auction. Furthermore, both approaches can be applied for all or for each $\mathrm{QoS}_{l}$. The second possibility has the advantage to be able to differentiate between QoS classes, e.g., the user can differentiate how to spent money for a real-time QoS class for calls and for a best effort class whose budget constraint hence is lower based on the relaxed time constraints.

3) Utility for UL and DL: The utility is expressed by the utility function $\eta\left(\alpha_{l}^{x, y}, \cdot\right)$ and states the utility a user gets if data of a certain category $x$ will be transmitted with respect to the preference $\alpha_{l}^{x, y}$. The function clearly depends on the preference parameters [10]. The user can select between a 
risk averse, risk neutral and risk encouraging behavior. Normally, the behavior regarding the critical data will be risk-neutral, because each data will be equally important to fulfill the QoS. Additionally, the function for uncritical data will generally be risk neutral or risk averse, but the different utility will be clearly less for each uncritical data than for a critical data of the same QoS classes, because of the QoS fulfillment. Thus the utility $\eta$ is a concave function over the data of the QoS buffer $\operatorname{QoS}_{l}$.

\section{Inter Data GREC Mapping}

Generally, all bid vectors bid $_{i}$ for each $\mathrm{RAT}_{i}$ can be commonly calculated to optimize the data transfer with respect to the utility $\eta$, the preference $\alpha$, cost constraint $\mathbf{c}$, data $D_{l}$ in the QoS-buffers $Q o S_{l}$ and the EV properties. This function inherently includes the IDGM and all $\mathrm{BIS}_{i}$. The computational effort would be tremendous to solve such a nonlinear optimization problem. Therefore, the tasks are separated into a global part realized in IDGM and local parts $\mathrm{BIS}_{i}$ for each $\mathrm{RAT}_{i}$ including the bidding strategy. The bidding strategy is independent of the RAT. The IDGM instructs the BIS $_{i}$ to bid for a certain number $N_{i}$ of $R R G_{i}$. Thus, the BIS can simply be instances of one algorithm.

The IDGM is designed to solve three main tasks which will be described in Sections VI-D.1, VI-D.2 and VI-D.3:

- The data $D_{l}$ in the QoS-buffer $Q o S_{l}$ has to be categorized depending on the user's needs and QoS criteria.

- The data $D_{l}$ in the QoS-buffer $Q o S_{l}$ has to be mapped to the number $N_{l, i}$ of $R R G_{i}$ needed for each $\mathrm{RAT}_{i}$. In turn, the inverse function assists in assigning data which will be transmitted to the RATs.

- The IDGM is responsible for allocating the data $D_{l}$ in the QoS-buffer $Q o S_{l}$ as good as possible to satisfy the user's needs resulting in an non-linear optimization problem in general.

1) Data Categorization (DC): One of the main tasks of IDGM is to categorize the data which are in the QoS buffer $Q o S_{l}$ in order to decide the number $N_{l, i}^{x}$ of $\mathrm{RRG}_{i}$ needed per $\mathrm{RAT}_{i}$ and per data category $x \in$ $\left\{c r i, u c r i i_{1}, \ldots, u c r i i_{J}\right\}$. The data is distinguished between critical data $D^{\text {cri }}$ which should be urgently sent in this auction period $T$ in order to fulfill the QoS criteria and uncritical data $D^{u c r i}$. If $D^{u c r i}$ is not sent, the QoS is not injured during $T$. The uncritical classes can be subdivided into prioritized subclasses $D^{u c r i i_{j}}$ depending on the urgency to send them in advance to fulfill the QoS $l$ and avoid high traffic time. This Data Categorizing Function (DCF) is situated in the Inter-MAC in order to categorize the UL data $D_{l}^{x, U L}$ and also in the RNC to classify the DL data $D_{l}^{x, D L}$. The information about the number of data $D_{l}^{x, y}$ for each category $x$, QoS class $l$ and each link $y$ is conveyed to the Inter RRG Allocation (IRA).
In principle, the user is self-responsible for the QoS, thus he can individually adjust the QoS criteria and parameters, even different for UL and DL. Originally, the operator has designed the network regarding the services to be offered with a certain QoS. The system should clearly be capable to transport at least the critical data $D^{\text {cri }}$. If the network is not overloaded and the user has stronger/weaker QoS criteria resulting in higher/lower number of critical RRG, the BIS will bid higher/lower leading to higher cost/data loss. For this reason the user should accept the QoS parameter the network was designed for, because he can also express its preferences through the UPM by choosing the proper QoS class.

2) Data GREC Mapping: The Data GREC Mapping (DGM) converts an amount of data $D_{i}$ to the $R R G_{i}$ needed to send this data with the $\mathrm{RAT}_{i}$. The transformation function $f(\cdot)$ depends on the RAT properties and channel characteristics which represent the EV. The RAT properties are mainly Channel Coding (CC), Modulation (MOD) and MAC/PHY Control Overhead (CO). Further attributes can specify the QoS classes and their mandatory RRG needs. Some RAT properties can also be dependent on the EV, like CC and MOD which can be adjusted with respect to the Signal to Noise and Interference Ratio (SNIR). The EV can adequately be described by the SNIR. Thus, the transformation for $R A T_{i}$ can be described as follows:

$$
\begin{aligned}
N_{i} & =f_{i}(\text { RAT properties, EV properties, } D) \\
& \approx f_{i}(C C, M O D, C O, S N I R, D)
\end{aligned}
$$

$f(\cdot)$ can be approximated based on past observations of the data transmission. Considering simple RATs it can also be envisaged that there exists an analytical expression, e.g., calculating BER from SNR, CO and MOD. The number $N_{i}$ of $R R G_{i}$ is an estimation because of the unknown and random EV.

For the computation of the RRG allocation to the RATs in the IRA and the allocation of data to the RATs after having gained GRECs for the specific RAT the inverse transformation $f^{-1}$ is necessary:

$$
\begin{aligned}
D & =f_{i}^{-1}\left(\text { RAT properties, EV properties, } N_{i}\right)(2) \\
& \approx f_{i}^{-1}\left(C C, M O D, C O, S N I R, N_{i}\right)
\end{aligned}
$$

The inverse transformation describes the estimated amount of data which may be transmitted assuming $N_{i}$ RRG.

3) Inter RRG Allocation: The Inter RRG Allocation (IRA) has to assign the BISs the number $N_{l, i}^{x, y}$ of RRG in order to fulfill the user's needs which can be separated into two different goals:

G1) Favoring the QoS, the QoS measure $\mu\left(Q o S_{l}\right)$ should be maximized and ideally the QoS should always be fulfilled. Thus, the critical data $D_{l}^{c r i}$ will be prioritized. The allocation algorithm can be logically separated into two parts: first the allocation of the critical data and then the uncritical data, because it is mandatory to send critical data instead of uncritical data in order to maximize $\mu\left(Q \circ S_{l}\right)$. Regardless, if 
the utility of uncritical data would be higher than the one of critical data, the allocation algorithm will firstly assign the critical data to the RATs and the IGM transforms it to $\mathrm{RRG}_{i}$ in order to maximize the user's needs with respect to minimal costs. Data in a category which has no preferences at all $(\alpha=0)$ will be neglected. In the second part, the same instance of the algorithm used for the critical data can be applied to the uncritical data. The algorithm also aims at maximizing the utility and chooses the economically best solution.

G2) In a different approach only the utility will be maximized regardless of the QoS. There is no preference for critical data. The algorithm does not distinguish between critical and uncritical data. Therefore the algorithm is not separated into different parts and the main parameters are the utility function $\eta_{l}^{x, y}$ and the preference parameters $\alpha_{l}^{x, y}$.

To decide which BIS ${ }_{i}$ should bid for how many $\mathrm{RRG}_{i}$, the IRA need information from each $\mathrm{BIS}_{i}$ about the techno-economical environment, specifically:

i) the efficiency $\rho_{l, i}^{x, y}$ of the $\mathrm{BIS}_{i}$ which is defined as the ratio of the number $N_{l, i}^{x, y, w}$ of RRG won to the number $N_{l, i}^{x, y, b}$ of RRG bidden:

$$
\rho_{l, i}^{x, y}=\frac{N_{l, i}^{x, y, w}}{N_{l, i}^{x, y, b} .}
$$

$\rho_{l, i}^{x, y}$ is a measure of how good the BIS works in the current $\mathrm{EV}$ and serves as a main parameter to decide whether this $\mathrm{BIS}_{i}$ could be bid for more/less $\mathrm{RRG}_{i}$ depending on high/low efficiency.

ii) the price $p_{l, i}^{x, y}$ payed for the $N_{l, i}^{x, y, w}$ RRG won. The IRA divides this price by the actual data $D_{l, i}^{x, y, s}$ sent in order to get a measure $\delta_{l, i}^{x, y}$ of the cost per bit:

$$
\delta_{l, i}^{x, y}=\frac{p_{l, i}^{x, y}}{D_{l, i}^{x, y, s}}
$$

iii) the maximal number $N_{i}^{y}$, the number $N_{i}^{y, w}$ won and the cumulative number $N_{i}^{y, c w}$ won of $\mathrm{RRG}_{i}$. The relationships between these figures are useful to predict the load of the cell $i$ and to determine the increment/decrement of the $N_{l, i}^{x, y, p} \mathrm{RRG}$ proposed to the BIS $_{i}$ by the IRA. For example, if $N_{i}^{y}-N_{i}^{y, c w}>$ 0 , i.e., in case of more supply than demand, the IRA will clearly prefer this RAT in comparison to another one assuming the other conditions are the same.

iv) the QoS classes supported by this RAT.

Moreover, the UPM provides the IRA with the preference parameters $\alpha_{l, i}^{x, y}$ which serve as parameters of the specific utility function $\eta_{l, i}^{x, y}\left(\alpha_{l, i}^{x, y}, \cdot\right)$. The DCs of both the UL and DL side convey the categorized data $D_{l, i}^{x, y}$. Following the aforementioned two goals, the satisfaction of the user's needs has to be quantized in order that the algorithm can optimize it. A measure of this satisfaction is defined as the quadratic utility error function $\Delta_{\eta}$ that is the quadratic difference of the utility expected $\eta_{l}^{x, y}\left(\alpha_{l}^{x, y}, D_{l, i}^{x, y, p}\right)$ resulting from the data $D_{l, i}^{x, y, p}$ proposed to the utility wanted $\eta_{l}^{x, y}\left(\alpha_{l}^{x, y}, D_{l, i}^{x, y, q}\right)$ [2] of the data $D_{l, i}^{x, y}$ in the QoS buffer $\operatorname{QoS}_{l}$ :

$$
\begin{aligned}
\Delta_{\eta}\left(D^{p}, D^{q}\right) & =\sum_{x, y, l}\left(\eta_{l}^{x, y}\left(\alpha_{l}^{x, y}, D_{l}^{x, y, p}\right)\right. \\
& \left.-\eta_{l}^{x, y}\left(\alpha_{l}^{x, y}, D_{l}^{x, y, q}\right)\right)^{2} .
\end{aligned}
$$

The minimization of eq. (5) leads to the highest satisfaction of the user's needs with respect to cost and to other users' needs as well. If a user can send more data $D_{l}^{x, y}$ than wanted regarding a specific QoS class, this additional purchase power could be saved. Moreover, if every user takes more RRG than wanted, the price will increase, thus an equilibrium exists when all users aspire to the utility wanted and not maximize the utility.

To write the algorithm in a more overall compact manner an additional description is needed. The function $g$ maps all the input to the proposed RRG allocation $N_{l, i}^{x, y, p}$ :

$$
g\left(x, y, l, i, D_{l}^{x, y}, B I S P R\right)=\left(N_{l, i}^{x, y, p}\right),
$$

whereas the $\mathrm{BIS}_{i}$ properties are described by the 5-tuple:

$$
B I S P R=\left(\rho_{l, i}^{x, y}, \delta_{l, i}^{x, y}, N_{i}^{y}, N_{i}^{y, w}, N_{i}^{y, c w}\right) .
$$

Generally, there does not exist a closed analytical representation of $g$. In fact, $g$ has to be estimated and approximated.

Coming back to the goal opportunities G1 and G2, the algorithms can be written as an optimization problem with some side constraints (s.c.):

$$
\begin{aligned}
& \text { A1 } \operatorname{cost}^{c r i} \rightarrow \min \\
& \text { s.c. } \quad \Delta_{\eta}\left(D^{c r i, p}, D^{c r i, q}\right) \rightarrow \min \\
& \text { s.c. } \quad D_{l, i}^{c r i, y, p}=\sum_{i} f_{i}^{-1}\left(\cdot, N_{l, i}^{c r i, y, p}\right) \\
& \text { s.c. } g\left(D_{l}^{c r i, y}, B I S P R_{i}\right)=\left(N_{l, i}^{c r i, y, p}\right) \\
& \operatorname{cost}^{u c r i} \rightarrow \min \\
& \text { s.c. } \Delta_{\eta}\left(D^{u c r i, p}, D^{u c r i, q}\right) \rightarrow \min \\
& \text { s.c. } \quad D_{l, i}^{u c r i, y, p}=\sum_{i} f_{i}^{-1}\left(\cdot, N_{l, i}^{u c r i, y, p}\right) \\
& \text { s.c. } g\left(D_{l}^{u c r i, y}, B I S P R_{i}\right)=\left(N_{l, i}^{u c r i, y, p}\right) \text {. }
\end{aligned}
$$

At first, the algorithm will minimize $\Delta_{\eta}$ which is the side condition (s.c.) to the cost minimization with respect to the critical data, that is, the minimization of $\Delta_{\eta}$ constricts the solution set of the cost minimization. The second part is the same algorithm for the uncritical data regarding the solution of the first part as side constraint.

Dropping the aspiration to fulfill the QoS and act purely to satisfy the user's needs, there is no preference for the data categorized based on the QoS parameters:

$$
\begin{aligned}
\mathrm{A} 2 \text { cost } & \rightarrow \min \\
\text { s.c. } & \Delta_{\eta}\left(D^{x, p}, D^{x, q}\right) \rightarrow \min \\
& \text { s.c. } \quad D_{l, i}^{c r i, y, p}=\sum_{i} f_{i}^{-1}\left(\cdot, N_{l, i}^{x, y, p}\right) \\
& \text { s.c. } \quad g\left(D_{l}^{x, y}, B I S P R_{i}\right)=\left(N_{l, i}^{x, y, p}\right)
\end{aligned}
$$


The optimal function $g_{\text {opt }}$ can be approximated by several Artificial Intelligences (AIs), like Bayesian learning based on experience or knowledge based complex differential controller. For the last item, a possible realization of IRA is shown in the following.

The differential strategy looks only 2 steps back into the past, in order to exploit the tendency of the situation. The aim of this algorithm is to decide the data allocation per RAT based on his own information without using any information about the other users behavior, because their behavior are implicitly included in the own goods won depending on the bids. Moreover, based on the very shorttime repetition a strategy is proposed whose execution is very fast and linear in the number of bid strategies. The difficulties are to predict the market behavior, to evaluate the bidding strategy performance and finally to make a decision. Therefore, the algorithm can be divided into three steps:

S1 Based on the feedback of the bidding strategy, a recommendation will be given wether the bidding strategy is able to gain more data or not. The algorithm recommends for each QoS class $l$, link $y$ and category $x$. For the sake of simplicity these indices will be neglected in the following. The criteria are the tendencies of the efficiencies $\rho_{T}, \rho_{T-1}$ and data won $D_{i, T}, D_{i, T-1}$ for the past two auctions before $T$ and $T-1$. At first the difference of the data has to be computed

$$
\Delta D_{i, T}=D_{i, T}-D_{i, T-1} .
$$

A preliminary predecision which recommends the data allocation tendency $\Delta D$ will be calculated according to the following rules which can be seen as a defuzzying approach:

$$
\begin{aligned}
& \text { - } \rho_{T} \geq \rho_{T-1} \wedge \Delta D_{i, T} \geq 0 \Rightarrow \Delta D_{i}>0 \\
& \text { - } \rho_{T}<\rho_{T-1} \wedge \Delta D_{i, T} \geq 0 \Rightarrow \Delta D_{i}<0 \\
& \text { - } \rho_{T} \geq \rho_{T-1} \wedge \Delta D_{i, T}<0 \Rightarrow \Delta D_{i}<0 \\
& \text { - } \rho_{T}<\rho_{T-1} \wedge \Delta D_{i, T}<0 \Rightarrow \Delta D_{i}>0 \\
& \text { - } \rho_{T}>0 \wedge \rho_{T-1}=\text { nan } \Rightarrow \Delta D_{i}>0
\end{aligned}
$$

That is if there is no intention possible because the last but one efficiency is not available, but the bidding strategy gains RRGs, the bidding strategy will be awarded with a positive tendency, otherwise not.

- $\rho_{T}=0 \wedge \rho_{T-1}=$ nan $\Rightarrow \Delta D_{i}<0$

- $\rho_{T}=$ nan $\Rightarrow \Delta D_{i}>0$

If there is no information about the last bidding strategy behavior, the algorithm assigns a positive tendency.

S2 After making this hard decision the second part will quantize the tendencies in a data incrementation/decrementation resulting in a data proposal $D_{\text {prop }, i}$. This can be seen as a consecutive soft decision. Taking into account a less computational, but efficient decision, the proposals will be determined without any loops.

- If a bidding strategy for RAT $i$ got a positive tendency $\Delta D_{i}>0$, the positive recommended amount of data $D_{\text {prop }, i}$ is a proportion $\delta_{\text {prop }}^{\text {pos }}$ of the difference between the maximum data $D_{\max , i}$ available for this RAT $i$ and the amount of data needed $D_{i, T}$ assigned in the last auction:

$$
D_{\text {prop }, i}=\left(D_{\max , i}-D_{i, T}\right) \cdot \delta_{\text {prop }}^{\text {pos }}+D_{i, T} .
$$

This is chosen, because the difference is a measure of the capacity which could be occupied by the user terminal. To explore this capacity, a fraction of this difference is added with respect of the unknowledge of the other bidders' market behaviors. Moreover, this expression converges to $D_{\max , i}$ assuming always gaining the proposed data $D_{i, T}$.

- If the recommended tendency is negative $D_{i}<$ 0 the proposed amount of data $D_{\text {prop }, i}$ is the data needed in the last auction reduced by a fractional amount described by the parameter $\delta_{\text {prop }}^{n e g}$ :

$$
D_{\text {prop }, i}=\left(1-\delta_{\text {prop }}^{n e g}\right) D_{i, T} .
$$

S3 The proposed amounts of data based on the soft decision reflect the relative weights to allocate the real amounts of data in order to avoid redundancy and consequently less utility and higher costs. Concludingly, the amounts of data the IRA assigns to the bidding strategies are, recalling that this is executed for all QoS classes $l$, links $y$ and categories $x$ categories $x$ :

$$
D_{i}=\frac{D_{\text {prop }, i}}{\sum_{i} D_{\text {prop }, i}} D
$$

\section{E. Bidding Strategy}

The $\mathrm{BIS}_{i}$ bids periodically for $\mathrm{RRG}_{i}$ of $\mathrm{RAT}_{i}$ in only one cell ${ }_{i}$ based on the argumentation in Section V. The bidding strategy is independent of the RAT, thus for each $\mathrm{RAT}_{i}$ instances of the same bidding algorithm can be applied. Nevertheless, the bidding strategy can clearly be different and could be modified or exchanged in order to improve the allocation if the performance is worse.

Normally, BISs of different RATs need not to exchange data, because based on the application diversity of the different RATs the ASEV will differ. But, if a UT is connected to more cells of the same RAT simultaneously, the ASEV can be quiet similar. Considering a soft handover scenario, the user will enter the new cell and the new BIS instance can use the past experience of the old neighborhood cell because of the continuous change.

1) Input: The bidding strategy needs information $I$ about the user's preferences $\alpha_{l}^{x, y}$, behavior $\eta_{l}^{x, y}$ and purchase power $\mathbf{c}_{l}^{x, y}$ provided by the UPM and the number $N^{n}$ of $\mathrm{RRG}_{i}^{n}$ needed and calculated by the IDGM. To incorporate the user's behavior into the BIS, the information should be available for all necessary QoS classes $\mathrm{QoS}_{l}$ and categories $x$ and can be expressed by the set $I$ :

$$
I=\left\{\left(\alpha_{l}^{x, y}, \eta_{l}^{x, y}, \mathbf{c}_{l}^{x, y}, N_{l}^{x, y}, l, x, y\right) \mid \forall l, x, y\right\}
$$


Besides this user-based information, the BIS also receives information about past auctions as long as the UT attends to a proper cell. Depending on the AM implementation the BIS only gets information about the outcome of the auction if it actively participates and not only listen. Furthermore, the allocation vector a broadcasted by the EM offers information about the auction result and transmission. In turn, the EM decides about the degree of information a UT gets from the other users, to calculate the bid vector $\mathbf{b i d}_{i}$.

After the computation, the BIS conveys the bid vector $\operatorname{bid}_{i}$ to the AM. The AM gets the information for which link $y$ and QoS-class $l$ the user wants to bid $b_{j}$ per $\mathrm{RRG}_{i}$ for the number $N_{l, j, i}^{x, y}$ :

$$
\operatorname{bid}_{i}=\times_{j}\left(N_{l, j, i}^{x, y}, b_{j, i}, x_{j, i}, l_{j, i}\right)
$$

2) Goals: The bidding strategy can be designed to fulfill two distinct goals, that is, A1) with and A2) without respect to the QoS similar to the IDGM goal opportunities:

$$
\begin{aligned}
& \text { A1) } \\
& \mathbf{b i d}^{p, c r i} \in \mathbf{S}_{1}^{c r i}=\left\{\mathbf{b i d} \mid \operatorname{argmin}_{\left.\mathbf{b i d} \in \mathbf{S}_{2}^{c r i} \operatorname{cost}\right\}}\right\} \\
& \text { s.c. } \quad \mathbf{S}_{2}^{c r i}=\left\{\mathbf{b i d} \mid \operatorname{argmin} \text { bid } \Delta_{\eta}\left(N^{c r i, w}, N^{n}\right)\right\} \\
& \text { s.c. budget constraint } \\
& \Downarrow \\
& \text { bid }^{p, u c r i} \in \mathbf{S}_{1}^{u c r i}=\left\{\mathbf{b i d} \mid \operatorname{argmin}_{\mathbf{b i d} \in \mathbf{S}_{2}^{u c r i} \operatorname{cost}}\right\} \\
& \text { s.c. } \quad \mathbf{S}_{2}^{u c r i}=\left\{\mathbf{b i d} \mid \operatorname{argmin}_{\mathbf{b i d} \in \mathbf{S}_{1}^{c r i}} \Delta_{\eta}\left(N^{u c r i, w}, N^{n}\right)\right\} \\
& \text { s.c. budget constraint } \\
& \Rightarrow \text { bid }=\text { bid }^{u c r i, p}
\end{aligned}
$$

Ideally, this first opportunity chooses the bid which minimizes the cost with respect to maximize the user's needs measured by $\Delta_{\eta}$ under the side constraint of QoS fulfillment and the budget constraint. The algorithm is divided in two parts. First the bid vector proposal bid $^{\text {cri,p }}$ will be calculated for the critical RRG, then the proposed bid vector bid $^{u c r i, p}$ regarding the uncritical RRG will be determined depending on the result of the first part, e.g. budget constraint. The bid vector $\mathbf{b i d}^{u c r i, p}$ is the final bid vector. For each part the bid will be computed which minimizes the cost with respect to maximize the user's needs under the side constraint of the budget constraint.

Without respect to the QoS, a bidding strategy does not need to prioritize the RRG which should carry the critical data. Thus, the algorithm is:

A2)

$$
\begin{aligned}
& \text { bid } \in \mathbf{S}_{1}=\left\{\mathbf{b i d} \mid \operatorname{argmin}_{\left.\mathbf{b i d} \in \mathbf{S}_{2} \operatorname{cost}\right\}}\right. \\
& \text { s.c. } \quad \mathbf{S}_{2}=\{\mathbf{b i d} \mid \operatorname{argmin} \\
&\text { bid } \left.\Delta_{\eta}\left(N^{w}, N^{b}\right)\right\} \\
& \text { s.c. } \quad \text { budget constraint }
\end{aligned}
$$

The budget constraint can be both an integral limit over time to combat price peaks and an upper bound for each bid depending on the QoS class $\mathrm{QoS}_{l}$ and category $x$. The last proposal is simpler to implement, but cannot react flexibly if temporarily the prices are higher than these bounds resulting in a worse QoS fulfillment.

The minimization of the quadratic utility error $\Delta_{\eta}$ of the $N^{w}$ RRG won (ideal) and $N^{e}$ estimated (real) and the $N^{n}$ RRG needed is the quantized maximization of the user's needs. But $N^{w}$ is a future information which is not available during the calculation, because the other bids bid $_{-j}$ are not known to bidder $j$. The auction algorithm $A$ has to be known to adjust and choose the most suitable BIS. Thus, if $\mathbf{b i d}_{-j}$ are known, $N^{w}=A_{j}\left(\mathbf{b i d}_{j}, \mathbf{b i d}_{-j}\right)$ is a determined function of all bids and the minimum of $\Delta_{\eta}$ can be calculated. Focusing on the multi-unit sealedbid auction only statistical parameters and functions of the other bids bid $_{-j}$ like histograms can be used to predict $N^{w}$ by $N^{e}$. Therefore, the minimization can only be reached by a bid vector with a certain probability.

Additionally, the bid bid $_{j}$ which minimizes the cost can only be determined if all bids are known. Facing the same problem as mentioned above, the ideal expression has to be modified to work with the uncertainty of the other bids. One opportunity is to choose this bid for which the maximum expected costs are minimal.

3) Algorithm Concepts: The optimization can fall back on history, experience and current data. Different AI methods can be applied like neuronal networks, fuzzy logic, Bayesian networks, generic algorithms or a combination of them [2]. To implement the right AI is the questions of computational power, memory and data available. In the following two concepts of possible bid strategies will be presented.

Bayes Optimal Classifier: The Bayes optimal classifier states, which classification bid of the new instance, i.e., auction, is most probable, given the data $D$ of past auctions [12]:

$$
\mathbf{b i d}^{o p t}=\arg \max _{\mathbf{b i d} \in B} \sum_{h_{j}} P\left(\mathbf{b i d} \mid h_{j}\right) P\left(h_{j} \mid D\right)
$$

whereas $h_{j}$ is a hypothesis of the target $\mathbf{b i d}^{\text {opt }} \in \mathbf{S}_{1}$.

An instance $\xi$ can be described by the 5-tuple:

$$
\xi=\left(\mathbf{b i d}_{-j}, N_{-j}^{w}, N_{j}^{w}, r, D S\right),
$$

including the other bids bid bij $_{-j}$, the $N_{-j}^{w}$ RRG won by the others, the $N_{j}^{w}$ RRG won by itself, the reserve price $r$ and the demand/supply information $D S$. If $\xi$ is completely known the $\mathbf{b i d}^{\text {opt }}=c(\xi)$ can be determined whereas $c(\xi)$ is the mapping of the ideal algorithm of the bidding strategy. The unawareness of the current bid and the corresponding RRG numbers, leads to an approximation of $c(\xi)$ by the hypothesis $h\left(N_{j}^{w}, r, D S\right)=\mathbf{b i d}^{p}$ which is bid $^{\text {opt }}$ for a certain probability

$$
P\left(\mathbf{b i d}^{p} \mid h\left(N_{j}^{w}, r, D S\right)\right)
$$

assuming $h\left(N_{j}^{w}, r, D S\right)$ the valid hypothesis. In order to get a measure of the correctness of $h\left(N_{j}^{w}, r, D S\right)$, the probability $P(h(\cdot, r, D S) \mid D)$ that the hypothesis is correct given $D$ has to be determined, e.g., if $h$ uses histograms based on $\mathrm{D}$, the accuracy has to be determined regarding the use as probability like $P\left(\mathbf{b i d}_{-j}\right)$.

The set of instances $\xi$ could be very large and sparse during the auction sequence. Thus, if there are new instances for which there is no experience, the information of the others should be weighted and applied to the new instances if possible. One example could be the interpolation or extrapolation of the histogram 
$H\left(\right.$ bid $\left._{-j} \mid r, D S\right)$. Therefore, an additional condition will be included in eq. (16):

$$
P_{m, c}=P\left(\mathbf{b i d}^{p} \mid h\left(N_{j}^{w, c}, r_{m}, D S_{m}\right),\left(N_{j}^{w, c}, r_{c}, D S_{c}\right)\right)
$$

with $N_{j}^{w, c}, r_{c}$ and $D S_{c}$ as the current parameters. Eq. (17) describes the probability, that $\mathbf{b i d}^{p}$ is the optimal bid using the hypothesis $h\left(N_{j}^{w}, r_{m}, D S_{m}\right)$, whereas the actually current parameters $\left(N_{j}^{w, c}, r_{c}, D S_{c}\right)$ differ from the hypothesis ones $\left(N_{j}^{w}, r_{m}, D S_{m}\right)$.

Finally, the Bayes Optimal Classifier in eq. (14) can be written as:

$$
\mathbf{b i d}^{\text {opt }}=\arg \max _{\mathbf{b i d} \in B} \sum_{r_{m}, D S_{m}} P_{m, c} P\left(h\left(\cdot, r_{m}, D S_{m}\right) \mid D\right)
$$

Expectation Classifier: The Expectation Classifier [2] replaces the $N^{w}$ by its expectation $N^{e}=E\left\{N^{w}\right\}$. Additionally the minimum of the cost in the worst case will lead to the bid $^{p}$ proposed:

$$
\begin{aligned}
& \mathbf{b i d}^{p, c r i} \in \mathbf{S}_{1}^{c r i}=\left\{\mathbf{b i d} \mid \operatorname{argmin}_{\mathbf{b i d} \in \mathbf{S}_{2}^{\text {cri }} \max \text { cost }}\right\} \\
& \text { s.c. } \quad \mathbf{S}_{2}^{c r i}=\left\{\mathbf{b i d} \mid \operatorname{argmin}_{\mathbf{b i d}} \Delta_{\eta}\left(N^{e, c r i}, N^{n}\right)\right\} \\
& \text { s.c. budget constraint } \\
& \Downarrow
\end{aligned}
$$

$\mathbf{b i d}^{p, u c r i} \in \mathbf{S}_{1}^{u c r i}=\left\{\mathbf{b i d} \mid \operatorname{argmin}_{\mathbf{b i d} \in \mathbf{S}_{2}^{u c r i} \max \operatorname{cost}}\right\}$

s.c. $\quad \mathbf{S}_{2}^{u c r i}=\left\{\mathbf{b i d} \mid \operatorname{argmin}_{\mathbf{b i d} \in \mathbf{S}_{1}^{c r i}} \Delta_{\eta}\left(N^{e, u c r i}, N^{n}\right)\right\}$

$$
\text { s.c. budget constraint }
$$

$\Rightarrow$ bid $=$ bid $^{u c r i, p}$

The hypothesis as defined include histograms which build upon $D$. If there is no value of the histogram available, a weighted extrapolation/interpolation will provide experience of other instances $\xi$ occurred.

The main difference between these two methods is either the decision according to the probability or the expectation.

\section{F. Economic Manager}

The EM is logically located in the MAC of each BS. Its functionalities should represent the operator's behavior. The main goal of an operator is to maximize his revenue which is reflected in the EM subentities RPC and AM. Based on the EM's location this system is spatially distributed. Normally, the EMs of different operators are not connected and work independently of each other, but it can also be imagined that EMs of the same operator collaborate which increases the revenue according to game theory [10]. The output of the EM, i.e., the allocation vector, is used to assign the users the resources and the transmission conditions, e.g., when to send at which channel.

The RPC is the leverage of the operator to influence the auction and the AM is the common known auction mechanism.

1) Reserve Price Calculator: The RPC is the same important entity comparable in AI, computational power and decision emphasize as the BIS. In comparison to the BIS, the RPC can elaborate on more historical information, because all bid vectors bid $_{j}$ and allocation vectors are accessible.
Its aim is to find this reserve price $r$ which maximizes the operators gain $G$. Ideally, this can be mathematically expressed by:

$$
r^{o p t} \in \mathbf{S}=\left\{r \mid \underset{r}{\operatorname{argmax}} G\left(j, B I S_{j}\left(I_{j}, r, D S\right), A\right)\right\},
$$

whereas $G$ depends on the action of the bidding strategies $B I S_{j}$ including the number $N^{n}$ of RRG wanted and the auction mechanism. Parts of this information are not available for the EM, because it is in future or not accessible for the EM. Thus the optimal solution in eq. (19) can only be approximated by exploiting past information. The approximation results in choosing the most appropriate hypotheses $h$ in the hypotheses space:

$$
r^{p}=h\left(t<t_{0}: I_{R P C, t}, A\right) .
$$

The inputs of the hypotheses are the kind of auction mechanism $A$ and the information triple $I_{R P C, t}$ at the time point $t$ including all bid vectors $\times_{j}$ bid $_{j}$ of each bidder $j$, the reserve price $r$, and the outcome of the auction mechanism $A\left(\times_{j} \mathbf{b i d}_{j}, r\right)$ :

$$
I_{R P C, t}=\left(\times_{j} \mathbf{b i d}_{j}, r, A\left(\times_{j} \mathbf{b i d}_{j}, r\right)\right) .
$$

In contrast to the BIS the RPC knows all bid, thus the prediction of the future bidding behavior can be better estimated.

Based on the approximation of $G$ from $h$ the outcome $r^{p}$ only belongs to the solution set $\mathbf{S}$ with a certain probability $P\left(h \mid t<t_{0}: I_{R P C, t}, A\right)$ that $\mathrm{h}$ is correct based on the experience of past auctions. Therefore, $r^{p}$ belongs to the fuzzy set $F$ described by $\left(\mathbf{S}, P\left(h \mid t<t_{0}: I_{R P C, t}, A\right)\right)$. This $r^{p}$ has to be chosen for which $P$ is maximal:

$$
r^{p}=\left\{r \in F \mid P\left(h \mid \forall t<t_{0}: I_{R P C, t}, A\right) \Rightarrow \max \right\}
$$

In [2] is a RPC proposed which possesses an integral and a differential part. The integral part is responsible for incorporating and for evaluating the importance of the experience. On the other hand, the differential part reacts on shortterm actions on the market. In this algorithm the hypothesis will be implicitly evaluated and selected from the proper hypothesis spaces.

2) Auction Mechanism: The AM should be designed to fulfill the high dynamic, repetition and minimum of signaling effort. As discussed in [13], the multi-unit sealed-bid auction is the most suitable regarding the open and sequential multi-unit sealed-bid auctions. These auctions belonging to the standard auctions can only be completely implemented into the system if the allocation is ideal, that is each RRG can be assigned to DL or UL independently of technical constraints. Otherwise, the allocation mechanism has to be adapted to these technical constraints in order to optimize the operator's gain leading to a no-standard, but RAT standard specific auction.

The EM offers multiple RRG which are auctioned simultaneously. These RRG can be identical or may have different evaluations, e.g., code auctioning in a CDMA system. For the sake of simplicity, the first method is 
valid in the following if nothing else is stated otherwise. The EM broadcasts the announcement of the auction and sends the reserve price $r$ and the number of RRG offered. The RAAs calculate the bid vectors and submit them to the EM. After collecting all bids, the AM calculates the RRG allocation and assigns the RAAs won the proper number of GRECs. First, assuming no joint radio resource optimization tool, the BS can only assign the UT RRG from its RRG whether the channel is disturbed or not. Second, if there is a joint radio resource optimization entity like JRRM in [11], GRECs interchanges between $\mathrm{BS}$ are possible under the premise that the operator does not reduce his revenue and the user gets an enhanced QoS. The allocation vector conveys to both the PHY and scheduler in UL and DL to adjust the transmission entities and to read out the data of the QoS buffers in UL and DL regarding the minimum utility error function.

\section{Simulation}

The whole system is implemented in a discrete event simulation tool. The environment consists of two different base stations $B S_{1}, B S_{2}$ and radio access technologies. For each cell an economical manager $E M_{1}$ and $E M_{2}$ is responsible for pricing, allocation and billing the cell capacity $C_{\max , 1}$ and $C_{\max , 2}$. The capacities are auctioned by multi-unit sealed-bid discriminatory auctions. The capacity of $B S_{1}$ is two times the capacity of $B S_{2}$. Both are clustered in RRGs, whereas each RRG can carry the same amount of data. For the sake of simplicity, but without loss of generality, the channels are ideal.

A certain number of clients $N_{c}$ attended both cells and all clients are capable for multi-homing, that is they are simultaneously connected to both base stations and participate in both auctions. The auctions within both cells occurs contemporarely and the auction repetition is the same time $T_{A}$. Each Radio Auction Agent possesses one instance of the same bidding strategy for each auction. This bidding strategy as proposed in [2] learns about the past by maintaining an histogram and approximately calculates statistical measure to determine a suitable bid vector which maximizes the fulfillment of the criteria. The utility functions are assumed to be linear resulting in a risk-averse user behavior by account both the critical and uncritical data. This involves that the scheduling algorithm and the DATA-GREC Mapping can be executed in linear time to gain an optimal assignment. The scheduler is responsible for reading out the data of the QoS buffers and send it down to the PHY, whereas the DGM maps the data of the QoS buffer into an optimal RRG constellation satisfying the bid protocol policy.

The QoS is characterized by two parameters, a minimum data rate $d_{m i n}$ and a minimum delay $\tau_{m i n}$. Two cases of traffic has been considered: real-time and packet traffic. There are two scenarios in which for each of them there are two QoS classes $Q o S_{1}$ and $Q o S_{2}$. In the first scenario both classes are real time traffic classes with incoming traffic data rate $d_{c, 1}$ and $d_{c, 2}$. In the second scenario the $Q o S_{1}$ traffic is realtime and the input of the $Q o S_{2}$ buffer are packets arriving according to a

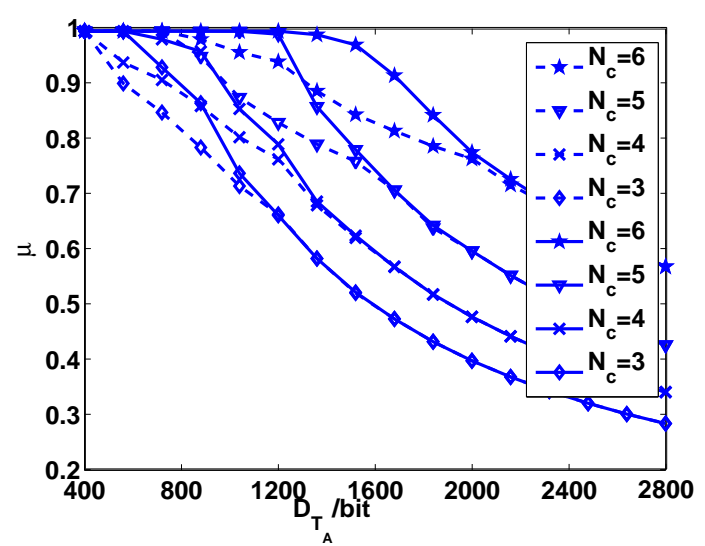

Fig. 3. Scenario 1: Comparison of the equal IRA (dashed line) and the proposed IRA (solid line)

POISSON process characterized by the averaged arrival time $\tau_{a}$ after sending a packet.

The proposed IRA algorithm which is responsible for the assignment of the resources to the bidding strategies is compared with the so-called equal IRA. The equal IRA divides the categorized data equiportional to the bidding strategies. This is the algorithm which does not exploit any information. In contrast to this, the proposed IRA tries to balance the load for the bidding strategy with respect of the maximum available capcity and the bidding startegy performance. It does not exploit explicit information of the other UTs market behavior.

For the following two scenarios, it is assumed that $E M_{1}$ offers $N_{\max , 1}=8 R R G$ clusters of $C_{\max , 1}$ and correspondingly $E M_{2} N_{\max , 2}=16 R R G$. Each cluster can carry $D_{R R G}=200 b i t$ resulting in a relative capacity $C_{\max , 1}$ and $C_{\max , 2}$ depending on the auction period $T_{A}$ :

$$
C_{\max , i}=\frac{N_{\max , i} \cdot D_{R R G}}{T_{A}}
$$

That is $B S_{1}$ can transmit at most 1600 bit per auction and $B S_{2}$ at most 3200bit.

\section{A. Scenario 1}

In this scenario there are certain number of clients $N_{c}$. The traffic into their QoS buffers is equal and the same as the minimum data rate of the $\operatorname{QoS}\left(d_{c, 1}=d_{c, 2}=d_{\text {min }}\right)$. The minimum delay is $1.75 T_{A}$. Consequently, after 2 auctions without sending the data the QoS is injured and the data will be removed from the buffers. The preferences $\alpha$ and the maximum costs $c_{\max }$ bit per bit are randomly choosen. The relation of the preferences $\alpha$ and maximum costs $c_{\max , b i t}$ are equivalent. Figure 3 shows the overall throughput $\mu$ defined as the cumulative data sent $D_{s}$ in relation to the cumulative data needed $D_{n}$ from the upper layer depending on the data needed $D_{T_{A}}$ per UT and per auction period $T_{A}$ for 3 to 6 clients attending the cells. For small $D_{T_{A}}$ the two allgorithms perform equal, because the load is as small as by equally dividing data to the $E M_{i}$ and the cells are not overloaded. The graph of the equal IRA starts to decrease faster 


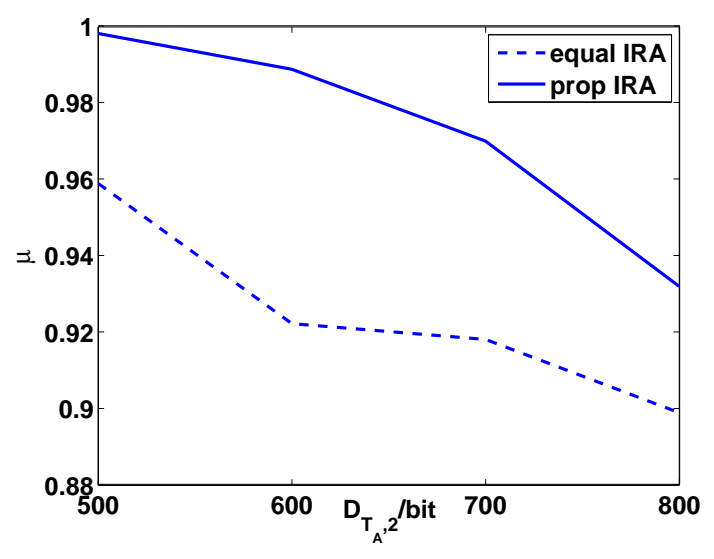

Fig. 4. Scenario 2: System behavior by mixed traffic

than the proposed IRA if the load is now more than $2 \cdot C_{\max , 1}$ and thus losses occur. In this state the balancing functionality of the proposed IRA takes effect resulting in an overall better capacity. Towards large $D_{T_{A}}$ the sum of the capacities of all UTs exceed the sum of $C_{\max , 1}$ and $C_{\max , 2}$, therefore the performance of both algorithm converges. For example, consider 4 user, then the equal IRA gets worse at $800 \mathrm{bit} / T_{A}$ and it approaches the graph of the proposed IRA at $1200 \mathrm{bit} / T_{A}$.

\section{B. Scenario 2}

4 users will attend the cells and the QoS buffers have different traffic. $Q o S_{1}$ gets real time traffic with $D_{T_{A}, 1}=400 b i t$ and data packtes arrive at $Q o S_{2}$ with the duration $T_{p}=5 T_{A}$ and the length of $D_{p}=400 \mathrm{bit} \cdot T_{p}$. The average arriving duration is exactly chosen to get in average $D_{T_{A}, 2}=400 b i t$. That is the same averaged load as for the constant rate case for which there is no performance difference of the algorithms. The mixed traffic as occuring for multi-service entities does not possess a smooth data rate over time, in fact it is bursty. This load variation lowers the performance of the equal IRA to the proposed IRA. Figure 4 shows again the overall throughput $\mu$ depending on the data needed $D_{T_{A}, 2}$ per UT, per auction duration and per $Q o S_{2}$. Besides the performance difference, the graphs decrease for larger $D_{T_{A}, 2}$ because if users send contemporarely, greater losses occur loss than for smaller $D_{T_{A}, 2}$ recalling the storing of uncritical data.

\section{CONCLUSION}

The awareness and cognition of a CR allows to give intelligent terminals responsibility to express the users needs, to fulfill the QoS and finally to satisfy the needs by evaluating the urgency to send the data. The multi-unit sealed-bid auction is suitable for short-term auctioning in communication systems and combines dynamic and local RRG pricing, allocation and billing allowing to apply the CR abilities.
A system proposal is described in which the RRG are priced, allocated and billed by a periodic auction sequence and CR abilities are applied. The UT possesses the opportunity to split the traffic over more than one RAT, thus services can be used by multi-homing.

\begin{tabular}{|c|c|}
\hline \multicolumn{2}{|r|}{$\begin{array}{c}\text { APPENDIX I } \\
\text { ABBREVIATIONS }\end{array}$} \\
\hline Abb. & Definition \\
\hline$\alpha$ & Preference parameter \\
\hline AI & Artificial Intelligence \\
\hline AM & Auction Mechanism \\
\hline $\mathrm{AS}$ & Auction Sequence \\
\hline ASEV & Auction Sequence EnVironment \\
\hline bid & Bid vector \\
\hline BIS & BIdding Strategy \\
\hline BISPR & BIS PRoperties \\
\hline $\mathrm{BS}$ & Base Station \\
\hline$\overline{\mathrm{CC}}$ & Channel Coding \\
\hline $\mathrm{CO}$ & MAC/PHY Control Overhead \\
\hline $\mathrm{CR}$ & Cognitive Radio \\
\hline$\delta$ & Price per bit \\
\hline$D$ & Amount of data \\
\hline $\mathrm{DCF}$ & Data Categorizing Function \\
\hline DGM & Data GREC Mapping \\
\hline$\eta$ & utility \\
\hline $\mathrm{AF}$ & Acting Function \\
\hline EM & Economic Manager \\
\hline EV & EnVironment \\
\hline$\overline{\text { FPM }}$ & Fixed Price Model \\
\hline GI & Gaining Information \\
\hline GREC & General Resource Elementary Credit \\
\hline $\bar{i}$ & Index of RAT \\
\hline IDGM & Inter Data GREC Mapping \\
\hline IMAC & Inter MAC \\
\hline IRA & Inter RRG Allocation \\
\hline$j$ & Index of user \\
\hline JRRM & Joint Radio Resource Management \\
\hline$l$ & Index of the $l^{t h}$ QoS class \\
\hline $\mathrm{LP}$ & Learning Process \\
\hline MAC & Medium Access Control \\
\hline MOD & MODulation \\
\hline$N$ & Number of RRG \\
\hline$p$ & Price \\
\hline$\rho$ & BIS efficiency \\
\hline$r$ & Reserve price \\
\hline RAA & RRG Auction Agent \\
\hline RAT & Radio Access Technology \\
\hline $\mathrm{RPC}$ & Reserve Price Calculator \\
\hline RRG & Radio Resource Goods \\
\hline SNIR & Signal to Noise and Interference Ratio \\
\hline SUBEV & SUBEnVironment \\
\hline UPM & User Profile Manager \\
\hline UT & User Terminal \\
\hline UTID & UT IDentification \\
\hline$x$ & Index of data category \\
\hline$y$ & Index of link $\{U L, D L\}$ \\
\hline
\end{tabular}




\section{ACKNOWLEDGMENT}

This work is partially funded by the Commission of the European Communities, under the 6th Framework Program for Research and Technological Development, within the project End-to-End Reconfigurability $\left(E^{2} R\right)$. The authors would like to acknowledge the contributions of their colleagues from $\mathrm{E}^{2} \mathrm{R}$ consortium.

\section{REFERENCES}

[1] Kloeck C., Jaekel H., and Jondral F., "Dynamic and Local Combined Pricing, Allocation and Billing System with Cognitive Radios," IEEE DySPAN'05, Baltimore, USA, 2005.

[2] Kloeck C., Jaekel H., and Jondral F., “ Auction Sequence as a New Resource Allocation Mechanism," IEEE VTC Fall 05,2005.

[3] N Amitay, "Resource auction multiple access (rama): efficient method for fast resource assignment in decentralised wireless pcs," Electronics Letters, vol. 28, no. 8, pp. 799801, 1992.

[4] Bayer D. L. Nutt G. J., "Performance of csma/cd networks under combined voice and data loads," Communications IEEE Transactions on, vol. 30, no. 1, pp. 6-11, jan 1982.

[5] Greenstein L.J. Amitay N., "Resource auction multiple access (rama) in the cellular environment," Vehicular Technology, IEEE Transactions on, vol. 43, no. 4, pp. 1101-1111, nov 1994.

[6] Nanda S. Amitay N., "Resource auction multiple access (rama) for statistical multiplexing of speech in wireless pcs," Vehicular Technology, IEEE Transactions on, vol. 43, no. 3, pp. 584-596, aug 1994.

[7] Roberto Boisson de Marca J. Javier Santivanez G., "Drama: A new deterministic mac protocol for wireless multimedia communications," IEEE to be completed, pp. 1043-1048, 1997.

[8] Amstrong M., "Optimal Multi-Object Auction," Economic Studies, vol. 67, pp. 455-481, 2000.

[9] ET Docket No. 03-108, " Noticeof Proposed Rule Making And Order," 2003.

[10] R.B. Myerson, Game Theory, Harvard University Press, 1997.

[11] Perez-Romero J. Giupponi L. Agusti R., Salient O., “ A fuzzy-neural based approach for joint radio resource management in a beyond 3G framework," Quality of Service in Heterogeneous Wired/Wireless Networks, 2004.

[12] T.M. Mitchell, Machine Learning, McGraw-Hill International Editions, 1997.

[13] Kloeck C., Jaekel H., and Jondral F., “ Auction Sequence as a New Spectrum Allocation Mechanism," IST Summit 05,2005 .

Clemens Kloeck (S'03) received the Dipl.-Ing. degree in electrical engineering from the Universitaet Karslruhe (TH) in 2002 and is currently working towards his $\mathrm{Ph}$. D. degree in dynamic and distributed wireless communication systems at the Universitaet Karlsruhe $(\mathrm{TH})$. He works within the End-to-End Reconfigurability $\left(\mathrm{E}^{2} \mathrm{R}\right)$ project in direction of Cognitive Radio, Cognitive Networks and dynamic spectrum sharing.

Holger Jaekel received a Diploma degree in applied mathematics (Dipl.-Math. techn.) in 1999 and a Doctoral degree in Electronic Engineering and Information Technology
(Dr.-Ing.) in 2003, both from the Universitaet Karlsruhe (TH). From 1999 to 2003 he worked as a research associate. Since 2003 he has been a senior researcher within the Communications Engineering Laboratory. His current research interests are in ultra wideband communications, mobile communications, signal processing in communications and dynamic spectrum allocation. Currently Dr. Jaekel is involved in several projects, focussed on but not restricted to ultra wideband communications.

Friedrich K. Jondral (SM'94) received Dipl.-Math. and Dr. rer. nat. degrees in mathematics from the Technical University of Braunschweig (Germany), in 1975 and 1979, respectively.

From 1979 to 1992 he was with AEG-Telefunken (now European Aeronautic Defence and Space Company, EADS), Ulm (Germany), where he held various reseach, development, and management positions. During this time he also lectured on courses in applied mathematics at the University of Ulm, where he was appointed Professor in 1991. Since 1993 he has been Full Professor and Head of the Communications Engineering Lab at the Universitaet Karlsruhe (Germany). His current research interests are in the fields of mobile communication, spread spectrum, signal analysis, pattern recognition, network capacity optimization and dynamic channel allocation. 